Available online at GSC Online Press Directory

GSC Biological and Pharmaceutical Sciences

e-ISSN: 2581-3250, CODEN (USA): GBPSC2

Journal homepage: https://www.gsconlinepress.com/journals/gscbps

(RESEARCH ARTICLE)

\title{
Malignant spiradenoma in a patient with Recklinghausen syndrome: a potential association
}

\author{
Stefania Erra *, Corinna Pizio and Ennio Nano \\ Surgical Pathology Department, Santo Spirito Hospital, Viale Giolitti 2, Casale Monferrato, Italy.
}

Publication history: Received on 20 September 2020; revised on 29 September 2020; accepted on 30 September 2020

Article DOI: https://doi.org/10.30574/gscbps.2020.12.3.0306

\begin{abstract}
Recklinghausen syndrome or neurofibromatosis type 1(NF1) is a hereditary condition transmitted with a dominant autosomal inheritance pattern. It's a systemic disorder characterized by involvements of skin, nerves, bone, soft tissues, iris and nervous system. Specifically a mutation of one allele of the oncosoppressor gene NF1 is the cause of the syndrome. Manifestations of neurofibromatosis include: benign tumors like neurofibromas, pheocromocytoma, juvenile xanthogranulomas, Lisch nodules, café-au-lait macules and freckles. Neurofibromatosis also correlates with an increased risk for malignant tumors like malignant melanoma, leukemia, brain gliomas. In this manuscript we report the association of NF1 with a malignant spiradenoma. A literature review has been made to investigate a possible correlation between these two entities.
\end{abstract}

Keywords: Neurofibromatosis type 1; Malignant spiradenoma; Neurofibromin; Ras-pathway; NF-kB pathway

\section{Introduction}

NF1 is a genetic syndrome caused by the loss of either alleles of the tumor suppressor gene NF1. It usually occurs in 1 on 3000/4000 people and has a high penetration. NF1 encodes for the protein neurofibromin, which production is sustained mostly by nerve cells and glial cells: oligodendrocytes and Schwann cells. Loss of function mutations of NF1 gene determine the deregulation of the cell cycle, leading to an unmitigated glial cell proliferation. Mutation of NF1 directly accounts for neurofibromas of peripheral nerves. They usually localize on or under the skin, with papule appearance; neurofibromas can also arise near the spinal cord or other visceral nerves. NF1 loss can also explain increased risk for optic nerve gliomas that can be asymptomatic or interfere with vision. Additional losses of other oncosoppressor genes and constitutional activation of oncogenes are responsible for carcinogenesis of malignant peripheral nerve sheath tumors, another condition associated with neurofibromatosis [1].

NF 1, typically, has an autosomal dominant pattern of inheritance and in $50 \%$ of the cases can be explained by acquisition of a mutated allele in the germinal lineage. These patients have a familiar history for neurofibromatosis. The other $50 \%$ of the cases are non-familiar as consequence of the acquisition of a new NF1 allele mutation in a large group of somatic cells. Knudson two-hit hypothesis can explain the pattern of inheritance of NF1 mutation.

Neurofibromatosis is characterized by a wide range of manifestations that vary between individuals. Some of them cannot be fully explained by loss of neurofibromin, like cafe-au-lait skin spots and dark freckles. Other abnormalities include: hypertension, short stature and macrocephaly, scoliosis, learning disabilities and deficit of attention and hyperactivity syndrome (ADHD) [2].

\footnotetext{
${ }^{*}$ Corresponding author: Stefania Erra

Surgical Pathology Department, Santo Spirito Hospital, Casale Monferrato, Viale Giolitti 2, Italy.

Copyright (C) 2020 Author(s) retain the copyright of this article. This article is published under the terms of the Creative Commons Attribution Liscense 4.0.
} 
Malignant eccrine spiradenomas are rare skin-adnexae tumors that arise from sweat-glands and can be subsides in two classes based on their behavior: low grade and high grade tumors. Only 50 cases have been described in the literature since 1972; the etiology is not known but there could be a correlation with sun exposure and immune suppression. They frequently grow from pre-existent benign eccrine spiradenomas and sometimes as primary tumors. The lesion can manifest clinically as a rapidly-growing and painful mass, sometimes ulcerated and bleeding, associated with redness of the skin and tenderness. Typically the growth is reported in the site of a pre-existent nodule. In $87.5 \%$ of the patients, it has been reported that the previously stable tumor has at least 2 years of age. Frequent localizations of the neoplasm include abdomen, limbs, trunk; uncommon locations are head and neck.

Microscopically primary tumors are composed of nest or aggregates of basaloid cells with scant cytoplasm and dense chromatin. Nuclei may show mild to moderate atypia; high mitotic activity and foci of necrosis are also typical findings. In tumor lesions, one cell population shows key features of benign eccrine spiradenoma, from which the malignant spiradenoma arises. These features comprehend: nest of basaloid or pale cells, with vesicular nuclei and pale cytoplasm, tubular structures with double epithelial lining [3].

The first treatment line for malignant spiradenomas includes traditional surgery technique like wide local excision or modern techniques like Mohs micrographic surgery. Surgery is very effective to treat localized disease, yielding a $100 \%$ disease-free survival rate at 33 month from initial diagnosis. Although this type of tumor tends not to be aggressive, in literature are reported cases of local recurrence, proximal lymph nodes involvement and distant metastatic disease. Adjuvant therapy, like radiotherapy and chemotherapy showed lack of efficacy in treating advanced disease, making necessary additional surgery. Mean survival for advanced metastatic disease was 16 months and a comparison in survival curves between surgery group and surgery group with adjuvant therapy showed no significant difference [4] [5].

\section{Case report}

Here we report a case of $60 \mathrm{y} / \mathrm{o}$ man, presenting to the dermatologic clinic of our institution, aware of a sudden growth of an ulcerated mass located in the hair cutis the previous two months.

At gross inspection the lesion appeared elevated, greyish, and entirely occupied the excision sample. It covered an area of $3 \times 2.5 \mathrm{~cm}$. Microscopically the lesion was composed of dermal circumscribed nest of monomorphic basaloid cells with dense chromatin and scant cytoplasm. Near the ulceration site in the epidermis, the neoplasm arranged in loosely cohesive sheets of cells with foci of necrosis [fig 1,2]. Vascular and neural invasion was not seen. Immunohistochemical profile of the tumor (CK8/18+, CK20-, p53+, CEA-) confirmed its skin-adnexa origin and Ki67 assessed its mitotic activity that scored an average value of $50 \%$. This last finding suggested a final diagnosis of low-grade malignant spiradenoma.

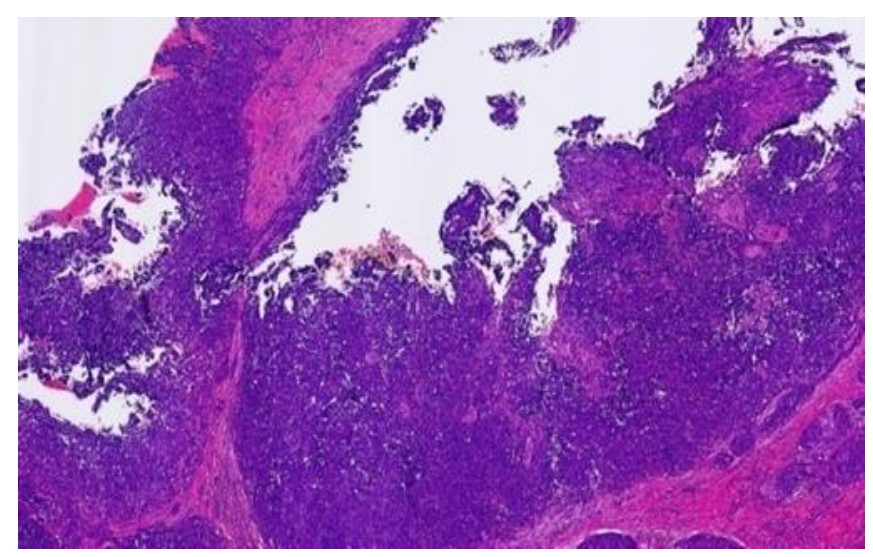

Figure $1 \mathrm{H} \& \mathrm{E}$ slide of the ulcerated portion of Malignant Spiradenoma. (Magnification 4x) 


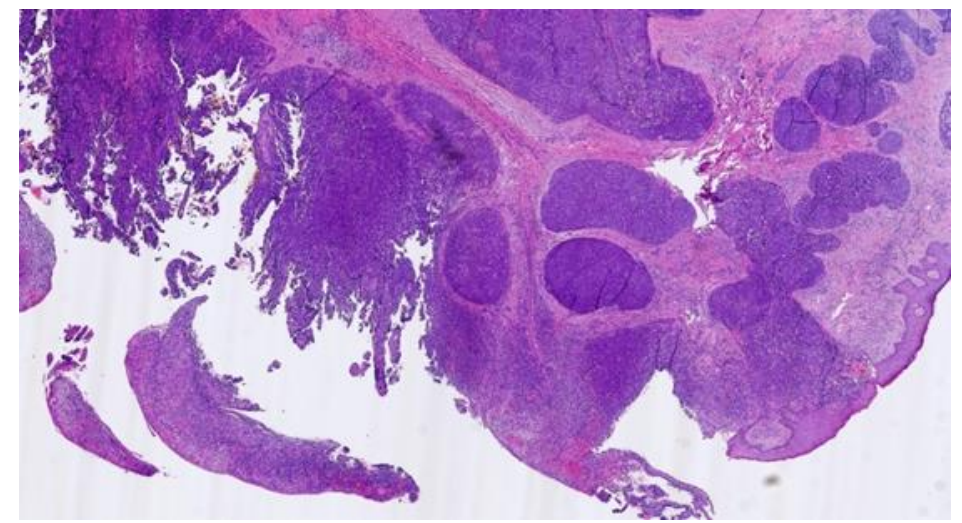

Figure 2 Well circumscribed nest and discohesive sheets (visible near the ulcerated margin) of monomorphic basaloid cells with dense chromatin. (Magnification 4x)

\section{Discussion}

NF1 is an autosomal dominant disease characterized by a high penetrance of complex and cellular neurofibromas and malignant peripheral nerve sheath tumor. Inheritance of one mutated allele of the NF1 gene and loss of heterozigosity (loss of the second allele) in a Schwann cell or its precursor facilitates tumorigenesis of neurofibromas. NF1 encodes for neurofibromin, a 320 KDa protein with a central catalytic Ras GAP called GAP related domain (GRD) that increase the hydrolisis and so the inactivation of RAS gene product. Neurofibromin is an oncosoppressor and a down-regulator of RAS/MAPK signaling cascade, which is normally involved in cell proliferation. Although this tumor suppressor gene (TSG) localizes in the cytoplasm, it contains a nuclear localization signal domain that suggests neurofibromin playing an unknown role in the nucleus. Neurofibromin is ubiquitously expressed among cell types but its degree of expression depends on developmental stage of the organism and cell type. Highest expression level is reached in adult neurons, Schwann cells, astrocytes, leukocytes and oligodendrocytes. Differential expression of this TSG suggests its important role in embriogenesis and differentiation of cell-type derived from the neural crest. It may be possible that neurofibromin could play a role in the development of skin-adnexa, which share a common ectodermic origin with nervous cell types. This hypothesis could sustain the incidental association of NF1 with eccrine spiradenomas and related tumor-adnexa [6].

Additional somatic mutations are needed to complete the process of tumorigenesis in neurofibromas and malignant peripheral tumor of nerve (MPNST) with loss of function of INK4a/ARF genetic locus and p53 protein and constitutional activation of oncogenes like RAS and RAF above all in cases with malignant transformations. Tumorigenesis is also sustained by an abnormal increased level of growth factors in the tumor environment. Heterozygous and haploid insufficient NF1 mast cells are involved in the aberrant production of growth factors. Loss of NF1 is also induced to alter mTOR activity [7] [8].

An NF1 loss association with spiradenoma, malignant spiradenoma and related tumors, cylindromas and hidradenomas is not described in literature. A heterogeneity of genetic signatures are thought to drive the tumor-adnexa group oncogenesis but are not specific for these type of tumors.

Some possible molecular mechanisms are described through three most common intracellular pathways. ALPK-1 gene encodes for a member of alfa-kinase protein family, located on chromosome 4q25 which is found mutated in different skin tumors, but also colon and lung cancer. This kinase protein is an activator of nuclear kappa factor complex (NF-kB) that acts as an oncogene after acquiring somatic mutation in the kinase domain. Its mutation was assessed equally for spiradenoma and spiradenocarcinomas, suggesting an early role in tumorigenesis. CYLD gene encodes a cytoplasmic protein with a cytoskeletal associated protein-glycine-conserved domain. It's a deubiquitinating enzyme that deregulates NF-kB pathway acting as aTSG. Germinal mutation of CYLD is associated with Brooke Spiegler syndrome, which manifests with cylindromatosis, cylindroma, trichoepithelioma and spiradenomas. c-MYB gene encodes for a transcription factor involved in positive regulation of cell-cycle. In a small percentage of spiradenomas and cylindromas, $\mathrm{c}$-myb is constitutively expressed as a result of a translocation between $\mathrm{q}$ and $\mathrm{p}$ arms of chromosome 6 and 9 respectively [9]. MYB-NFIB fusion gene is the resulting constitutively active chimeric gene. In spiradenomas the traslocation is not present, however c-myb expression is increased and stronger in spiradenocarcinoma. It's important to note that c-myb is positively regulated by NF-kB. Other mutations that characterized adnexa-tumor interest PTEN and p53 gene products [9] [10]. 
This little review of the common genetic hallmark of adnexa tumors speculates a major role of the NF-kB pathway in their oncogenesis; consequently NF1 could play only a minor role in this process. Although in literature there are documented case of different epithelial tumors concurrent with neurofibromatosis, like melanomas, hidradenoma and glomus tumors, statistical studies on their incidence in NF1 are inconclusive [11].

\section{Conclusion}

Malignant spiradenoma is a rare condition that only recently raised interest under the somatic mutations profile. Concurrency of this neoplasm with neurofibromatosis is only one of the several associations between a genetic disorder and a wide variety of skin disorders described in the literature. Even if an increased incidence of developing epithelial tumors in patients with neurofibromatosis has been reported, molecular mechanisms responsible of spiroadenocarcinoma in NF-1 is almost unclear. The lack of specificity in cancerogenetic molecular mechanism of skin adnexa can't support the hypothesis regarding a role of neurofibromin in these tumor hystotypes. In this reported case the rarity of the concurrency of malignant spiradenoma and neurofibromatosis implies that a correlation between the two entities might be not considered incidental.

\section{Compliance with ethical standards}

\section{Disclosure of conflict of interest}

Authors declare that there are no conflicts of interest in connection with this paper, and the material described is not under publication or consideration for publication elsewhere.

\section{Statement of informed consent}

Informed consent has been obtained from the subject included in the study.

\section{References}

[1] Arun D, Gutman DH. Recent advances in neurofibromatosis type 1.Curr Op in Neurol. 2004; 17(2): 101-5.

[2] Baralle D, Mattocks C, Kalidas K, Elmslie F, Whittaker J, Lees M, Ragge N, Patton MA, Winter RM, Constant C. Different mutations in the NF1 gene are associated with Neurofibromatosis-Noonan syndrome (NFNS). Am J Med Genet A. $119^{\circ} .2003$; (1): 1-8.

[3] Sami B, Ahmet K, Dinc S. Malignant eccrine spiradenoma on the lateral margin of nose as an infrequent localization. Indian J Dermatol. 2009; 54(2): 173-175.

[4] Andreoli MT, Itani KM. Malignant eccrine spiradenoma: a meta-analysis of reported cases. American Journal of Surgery. 2011; 201: 695-699.

[5] Naru RR, Inamdar MB, Das P, Kottu R. A rare case report of an aggressive malignant spiradenoma chest wall masquerading as carcinoma of unknown origin. J NTR Univ. Health Sci. 2017; 6:255-8.

[6] Jong WP, Sun N, Myung HK. A Case of eccrine Spiradenoma in a Patient with Neurofibromatosis. Ann Dermatol. 2010; 22(2): 191-193.

[7] Upadhyaya M, Cooper DN. Neurofibromatosis type 1: molecular and cellular biology. Springer Berlin Heidelberg. 2012.

[8] Oren NG, David HV, William TC. Neurofibromatosis Type 1 and tumorigenesis: molecular mechanisms and therapeutic implications. Neurosurg Focus. 2010; 28(1):E8.

[9] Rashid M, Van der Horst M, Mentzel T, et al. ALPK1 hotspot mutations a driver of human spiradenoma and spiradenocarcinoma. Nat, Commun. 2019; 10: 2-10.

[10] Michiel PJH, Zlatko M, Jason LH, Boštjan L, Thomas B. Morphologically low-grade spiradenocarcinoma: a clinic pathologic study of 19 cases with emphasis on outcome and MYB expression. Modern Pathology. 2015; 28: 944953.

[11] Wu H, Elenitsas R. Malignant nodular hidradenoma in a patient with neurofibromatosis type 1: A case report and review of the literature. Cutis. 2001; 68(4): 273-278. 


\section{Author's short biography}

\section{Stefania Erra}

Medical Doctor, specialized in Surgical Pathology and cytopathology. Senior hospital pathologist. Aziendal responsible of perinatal pathology. Aziendal responsible of cytopathology (FNAC pathology of breast, thyroid, salivary glands, gastroenteric mass, urinary pathology, thin prep slides). Aziendal reference for university students in preparing thesis degree. Aziendal tutor for universitary student in biology, medicine, and laboratory technicians. Cooperation with local university in preparing specialized courses for sanitaries. Educational activity towards university students with implementation of objective methods of learning. Teaching cytopathology in medical school university of Oriental Piedmont. Cooperation with regional sanitaries in redacting internal check list to manage oncologic pathology of head and neck region, uro-genital tract, breast cancer and gastroenteric ones. Authors of many publications (ORCID ID: 0000000254800383).

\section{Corinna Pizio}

Medical Doctor, resident in Surgical Pathology in medical school university of Turin. I'm interested in gastrointestinal pathology and skin pathology.

\section{Ennio Nano}

Doctor in biological sciences and student of Master degree in Biomolecular and Biomedical biology at the University of Piemonte Orientale. 